\title{
Molecular Markers and a Quality Trait Evaluation for Assessing the Genetic Diversity of Avocado Landraces from China
}

\author{
Yuanzheng Liu ${ }^{\dagger}, \mathrm{Yu} \mathrm{Ge}^{\dagger}{ }^{+}$, Rulin Zhan ${ }^{\dagger}$, Xinge Lin, Xiaoping Zang, Yanxia Li, Ying Yang and \\ Weihong Ma * \\ Haikou Experimental Station, Chinese Academy of Tropical Agricultural Sciences, Haikou 570102, China; \\ liuyuanzheng@catas.cn (Y.L.); geyu@catas.cn (Y.G.); zhanrulin@catas.cn (R.Z.); linxinge@catas.cn (X.L.); \\ zangxiaoping@catas.cn (X.Z.); liyanxia@catas.cn (Y.L.); yangying@catas.cn (Y.Y.) \\ * Correspondence: zjwhma@catas.cn; Tel.: +86-898-6677-3067 \\ + These authors contributed equally to this work.
}

Received: 27 February 2020; Accepted: 21 March 2020; Published: 2 April 2020

\begin{abstract}
Avocado is an economically important crop that is widely cultivated in the tropical and subtropical regions of China. However, there is a lack of sufficient information regarding the racial origins and genetic diversification of native avocado germplasm. On the basis of a race-specific kompetitive allele-specific PCR genotyping analysis, the 56 avocado accessions examined in this study were classified as complex hybrids, namely Guatemalan $\times$ Mexican $\times$ West Indian hybrids. The genetic relationships among the avocado accessions were assessed based on a quality trait evaluation and molecular characterization. The total flavonoid content was relatively useful for differentiating between native avocado accessions. A cluster analysis as well as principal component and coordinate analyses of quality traits and molecular characteristics differentiated the avocado accessions, but not according to geographical origins. Thus, the artificial flow of avocado germplasm or seed exchanges among farmers within specific regions may have contributed to the similar genetic backgrounds of avocado germplasm from different collections.
\end{abstract}

Keywords: Persea americana; phenotypic trait; EST-SSR marker; genetic relationship

\section{Introduction}

Avocado (Persea americana Mill.), which is a member of the family Lauraceae of the order Laurales, is one of the most economically important subtropical/tropical fruit crops worldwide [1-3]. Avocado is a highly diverse species with local variations that have resulted in various ecological races [4]. Additionally, it is native to Meso-America, but the 'subtropical' ecotypes (Guatemalan and Mexican) are currently widely grown in countries and regions with warm-to-cool subtropical and Mediterranean climates. The 'tropical' lowland ecotype (West Indian) is grown in genuinely tropical countries and warm subtropical regions [5]. These three ecotypes have been distinguished and identified based on genetic and morphological differences [6]. Avocado flowering exhibits a unique 'protogynous dichogamy' opening behavior, which favors cross-pollination, and sterility barriers do not exist between or among the three ecotypes [7]. Therefore, many commercial avocado accessions are often interracial hybrids of an uncertain ecotype, whether naturally occurring or induced artificially [8].

Avocados were first introduced to Taiwan province, China in 1918 [9], and have since been rapidly propagated to generate superior varieties for the commercial production of avocado plants with desirable characteristics and high-quality fruits. Since the late 1950s, hundreds of avocado varieties have been introduced to China from the United States of America, Israel, Mexico, and Central America [10,11]. Breeding and selection programs mainly at the Chinese Academy of Tropical Agricultural Sciences 
(CATAS), Guangxi Vocational and Technical College, and other state-owned or private farms have resulted in the selection of more than a dozen high-quality avocado varieties [10]. Moreover, natural hybridizations between avocado varieties often occur, producing many new avocado hybrids on state-owned or private farms. Several avocado accessions have also gradually developed in relatively isolated cultivation zones because of unique local environments [10].

Avocado is currently widely grown and sufficiently exploited in Hainan province in southern China, likely because the tropical climate and cultivation conditions in this province are ideal for avocado [12]. Baodao New Village, the Jianfeng private farm, the avocado germplasm resource garden affiliated with CATAS, and the Daling State Farm are four sites with many avocado landraces in Hainan province. In a previous study, the avocado landraces originating from the avocado germplasm resource garden of CATAS and the Daling State Farm were distinguished according to geographical origins based on our developed expressed sequence tag and simple sequence repeat (EST-SSR) markers [9,12]. Additionally, the avocado landraces were classified as Guatemalan $\times$ West Indian hybrids based on the race-specific kompetitive allele-specific PCR (KASP) genotyping results [12]. However, the avocado landraces from Baodao New Village and the Jianfeng private farm have not been evaluated and assessed regarding their genetic diversity and race.

Various types of molecular markers developed in previous studies have been applied to investigate the genetic diversity of global avocado resources, including random amplified polymorphic DNA [13], restriction fragment length polymorphisms [14], SSRs [8,15,16], and single nucleotide polymorphisms $[17,18]$. The consistency between morphological traits and molecular characteristics may be used to enable a highly accurate and largescale evaluation of genetic diversity [19]. To date, analyses of the diversity of avocado varieties based on agro-morphological traits and DNA polymorphisms have been described in only one report, in which 22 agro-morphological traits, 12 amplified fragment length polymorphism primer combinations, and 16 SSRs were used to estimate the genetic diversity of 23 avocado cultivars [20].

We previously generated race-specific markers from the specific length amplified fragment sequencing data of different avocado races to validate the racial origins of accessions with an unknown race [21]. Furthermore, highly polymorphic EST-SSR markers were generated from the transcriptomic data of various avocado races [22]. In the current study, we clarified the races of uncharacterized landraces and assessed the genetic diversity among avocado landraces based on quality traits and an EST-SSR marker system. The details regarding genetic diversity and races uncovered in this investigation may provide a solid foundation for managing the core germplasm collections, enabling genetic improvements, and broadening the genetic base of avocado materials used for breeding.

\section{Materials and Methods}

\subsection{Sample Collection}

To determine race and analyze the genetic diversity based on EST-SSR markers, 56 avocado accessions with an unknown race were obtained from the Nanfeng garden of the avocado germplasm resource (Danzhou, Hainan province, China; latitude $19^{\circ} 31^{\prime} \mathrm{N}$, longitude $109^{\circ} 34^{\prime} \mathrm{E}$, and $20 \mathrm{~m}$ above sea level), of which 46 (BDNV-1 to BDNV-46) were continuously collected in Baodao New Village (i.e., location of the CATAS headquarters) and 10 (JFPF-1 to JFPF-10) were continuously collected from the Jianfeng private farm (Ledong, Hainan province, China; latitude $18^{\circ} 50^{\prime} \mathrm{N}$, longitude $109^{\circ} 05^{\prime} \mathrm{E}$, and $50 \mathrm{~m}$ above sea level) since 2014. Additionally, 20 of these 56 avocado accessions that were fruit-bearing were selected for an evaluation of quality traits and an assessment of genetic diversity (Figure 1). For each accession, 10 mature fruits were randomly collected and promptly transported to our laboratory at $4-5{ }^{\circ} \mathrm{C}$. The pulp was separated from the fruits, homogenized, and stored at $4{ }^{\circ} \mathrm{C}$ until analyzed. All chemicals used in this study were of analytical grade and purchased from Sigma (Shanghai, China) and Sangon Biotech (Shanghai, China). 


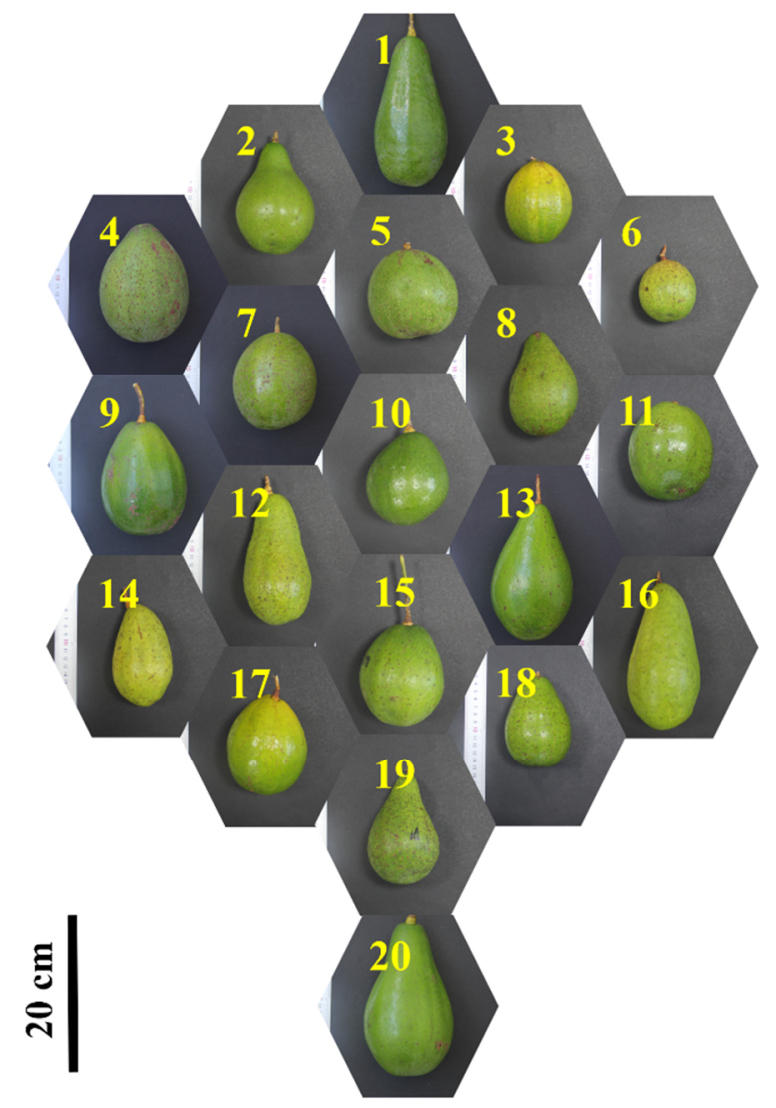

Figure 1. Appearance of 20 avocado samples selected for an evaluation of quality traits. 1: JFPF-1; 2: JFPF-2; 3: JFPF-3; 4: JFPF-4; 5: JFPF-5; 6: JFPF-7; 7: JFPF-8; 8: BDNV-2; 9: BDNV-3; 10: BDNV-4; 11: BDNV-6; 12: BDNV-8; 13: BDNV-11; 14: BDNV-12; 15: BDNV-14; 16: BDNV-15; 17: BDNV-21; 18: BDNV-29; 19: BDNV-35; 20: BDNV-37.

\subsection{DNA Extraction and Analysis of Avocado Accessions with an Unknown Race}

For each of the 56 analyzed avocado accessions, DNA was extracted from fresh leaves as previously described by Ge et al. [23]. To determine the races of these avocado accessions, six primers for race-specific single nucleotide polymorphism loci were used for KASP genotyping (Table S1) [21]. The KASP genotyping was completed as described by KBioscience (http://www.kbioscience.co.uk) and Ge et al. [21].

\subsection{EST-SSR Marker Analysis}

Sixteen previously developed EST-SSR primer pairs were used to analyze the genetic diversity of 56 avocado accessions with an unknown race [22]. The PCR amplification conditions were the same as those described by Ge et al. [23]. The amplified products were analyzed with the 96-capillary 3730x1 DNA Analyzer (Applied Biosystems, Foster City, CA, USA). The detection system included 8.9 $\mu \mathrm{L}$ HIDI (Applied Biosystems), $0.1 \mu \mathrm{L}$ LIZ (Applied Biosystems), and $1 \mu \mathrm{L}$ PCR products (1:10 dilution). A lack of amplification was considered indicative of a null allele.

\subsection{Quantification of Quality Traits}

\subsubsection{Moisture Assay}

The moisture content was evaluated as described by Ge et al. [24]. Briefly, fresh avocado pulp samples ( $5 \mathrm{~g}$ ) were dried and measured, after which their moisture content was calculated based on the differences between the fresh and dry weights. The moisture content data were expressed as g/100 g fresh weight. The measurements were performed with three replicates per accession. 


\subsubsection{Oil Content Assay}

The oil content was evaluated according to the method described by Ge et al. [24]. Briefly, avocado pulp samples were dried and transferred to a filter paper cylinder. Oil was extracted from the filtered solutions with the Soxhlet extractor. The extracts were then evaporated on a rotary evaporator and weighed. The oil content data were expressed as $\mathrm{g} / 100 \mathrm{~g}$ fresh weight. The experiments were performed in triplicate for each avocado accession.

\subsubsection{Soluble Sugar Assay}

The soluble sugar content was determined according to the colorimetric anthrone method described by Ge et al. [24]. The absorbance was measured at $620 \mathrm{~nm}$ with a spectrophotometer ( $1 \mathrm{~mL}$ distilled water and $5 \mathrm{~mL}$ anthrone reagent were mixed and used as the blank control). The soluble sugar content was calculated based on a calibration curve for glucose $\left(R^{2}=0.997\right)$ and expressed as $\mathrm{g} / 100 \mathrm{~g}$ fresh weight. All measurements were performed in triplicate for each accession.

\subsubsection{Soluble Protein Assay}

The soluble protein content was analyzed with the Coomassie Blue staining method described by Ge et al. [24]. The absorbance was measured at $595 \mathrm{~nm}$ with the Shimadzu UV-1800 spectrophotometer. Distilled water served as the blank control. The soluble protein content was calculated based on $0-100 \mu \mathrm{g} / \mathrm{mL}$ and $1000 \mu \mathrm{g} / \mathrm{mL}$ calibration curves prepared with bovine serum albumin $\left(R^{2}=0.997\right)$ and expressed as $\mathrm{mg} / 100 \mathrm{~g}$ fresh weight. All measurements were performed in triplicate for each accession.

\subsubsection{Ascorbic Acid Assay}

The ascorbic acid content was determined with a modified version of the 2,6-dichlorophenolindophenol method described by Ge et al. [24]. Briefly, fresh avocado pulp samples $(5 \mathrm{~g})$ were homogenized separately in $5 \mathrm{~mL}$ oxalic acid $(2 \%)$. The sample solutions were centrifuged, after which 10-mL aliquots of the extracts were titrated with 2,6-dichlorophenolindophenol. Oxalic acid ( $2 \%)$ was used as the blank control. Ascorbic acid was expressed as $\mathrm{mg} / 100 \mathrm{~g}$ fresh weight. The experiments were performed in triplicate.

\subsubsection{Total Phenol Assay}

The total phenol content was determined based on the method developed by Ge et al. [24]. The absorbance was measured at $760 \mathrm{~nm}$ with the Shimadzu UV-1800 spectrophotometer. Distilled water was used as the blank control. The total phenol content was calculated with a gallic acid calibration curve $\left(R^{2}=0.997\right)$ and recorded as gallic acid equivalents (mg GAE/100 g dry weight). The experiments were performed in triplicate for each accession.

\subsubsection{Total Flavonoid Assay}

The total flavonoid concentration was determined as described by Ge et al. [24]. The absorbance was measured at $510 \mathrm{~nm}$ with the Shimadzu UV-1800 spectrophotometer. Distilled water served as the blank control. The total flavonoid concentration was calculated with a rutin calibration curve $\left(R^{2}=0.995\right)$ and expressed as rutin equivalents (mg RE/100 g dry weight). The experiments were performed in triplicate.

\subsection{Data Analysis}

The number of observed alleles ( $\mathrm{Na}$ ), effective number of alleles $(\mathrm{Ne})$, observed heterozygosity (Ho), expected heterozygosity (He), and Shannon's information index (I) of each EST-SSR was assessed with the POPGEN program (version 1.32) [25]. The EST-SSR molecular investigation involved a cluster analysis of 56 accessions according to the unweighted pair-group method with arithmetic mean (UPGMA), which is a SAHN clustering procedure that compresses the patterns of variation 
into two-dimensional branch diagrams (dendrograms). A dendrogram was constructed according to Jaccard's formula with NTSYSpc (version 2.1) [26]. The principal component analysis (PCA) of 20 avocado accessions based on the seven quality traits and the principal coordinate analysis (PCoA) of 56 avocado accessions based on the 10 EST-SSR markers were performed with the MVSP program (version 3.21) (https://www.kovcomp.co.uk/mvsp/index.html). The seven quality traits were given as means \pm standard deviation. The data were analyzed using SPSS version 20.0 (SPSS Inc., Chicago, IL, USA). Significant differences among the seven quality traits of the 20 avocado accessions were verified by one-way analysis of variance, and Duncan's multiple comparison test was used to determine the statistical significance of differences between means at a 95\% confidence level.

\section{Results}

\subsection{Assignment of Avocado Races}

Six race-specific KASP markers were applied to determine the races of 56 avocado accessions with an unknown race. The KASP genotyping results revealed that all 56 accessions were complex hybrids, namely Guatemalan $\times$ Mexican $\times$ West Indian hybrids (Table S2).

\subsection{Characterization of Quality Traits}

There was a considerable range in the variability of the quality traits among 20 avocado accessions (Table 1). For the seven examined quality traits, the average coefficient of variation was $27.04 \%$. The lowest and highest coefficients of variation were $3.19 \%$ (moisture content) and $54.94 \%$ (total flavonoid content), respectively. The seven quality traits of 20 avocado accessions are provided in Table S3, and the significant differences in the seven quality traits were observed among most of the accessions $(p<0.05)$.

Table 1. Details regarding seven quality traits among 20 analyzed avocado accessions.

\begin{tabular}{cccccc}
\hline Quality Traits & Maximum & Minimum & Mean & $\begin{array}{c}\text { Standard } \\
\text { Deviation }\end{array}$ & $\begin{array}{c}\text { Coefficient of } \\
\text { Variation (\%) }\end{array}$ \\
\hline $\begin{array}{c}\text { Moisture } \\
\text { (g/100g) } \\
\text { Oil }\end{array}$ & 86.33 & 77.00 & 82.50 & 2.63 & 3.19 \\
$\begin{array}{c}(\mathrm{g} / 100 \mathrm{~g}) \\
\text { Soluble sugar } \\
\quad(\mathrm{g} / 100 \mathrm{~g})\end{array}$ & 11.64 & 5.44 & 8.01 & 1.56 & 19.53 \\
$\begin{array}{c}\text { Soluble protein } \\
(\mathrm{mg} / 100 \mathrm{~g})\end{array}$ & 1.44 & 0.76 & 1.12 & 0.22 & 19.77 \\
$\begin{array}{c}\text { Ascorbic acid } \\
(\mathrm{mg} / 100 \mathrm{~g})\end{array}$ & 6.56 & 1.11 & 2.19 & 0.61 & 28.08 \\
$\begin{array}{c}\text { Total phenol } \\
(\mathrm{mg} / 100 \mathrm{~g})\end{array}$ & 8.92 & 12.75 & 40.83 & 13.75 & 33.68 \\
$\begin{array}{c}\text { Total flavonoid } \\
(\mathrm{mg} / 100 \mathrm{~m})\end{array}$ & 9.12 & 3.27 & 6.21 & 1.87 & 30.05 \\
\hline
\end{tabular}

A cluster analysis grouped the 20 examined accessions into two major sections (Figure 2). Consistent with the results of the evaluation of quality traits, a clear sub-clustering based on the total flavonoid content was observed. Section I comprised accessions with high total flavonoid contents, including three accessions originating from Baodao New Village. Section II consisted of 17 accessions with low total flavonoid contents, of which 10 originated from Baodao New Village and seven originated from the Jianfeng private farm. Additionally, the oil content was closely related to the ascorbic acid, total phenol, and total flavonoid contents, whereas the remaining quality traits, including moisture, soluble sugar, and soluble protein contents were grouped together. Figure S1 presents the distribution of 20 avocado accessions according to the first principal components of a PCA. On the 
basis of the first principal component, which accounted for $37.03 \%$ of the total variation, the accessions were generally distributed in two groups. The avocado accessions with high and low total flavonoid contents were basically grouped separately. The second principal component accounted for $25.65 \%$ of the total variation. Finally, the native avocado accessions were generally grouped according to their total flavonoid content rather than their geographical origin.

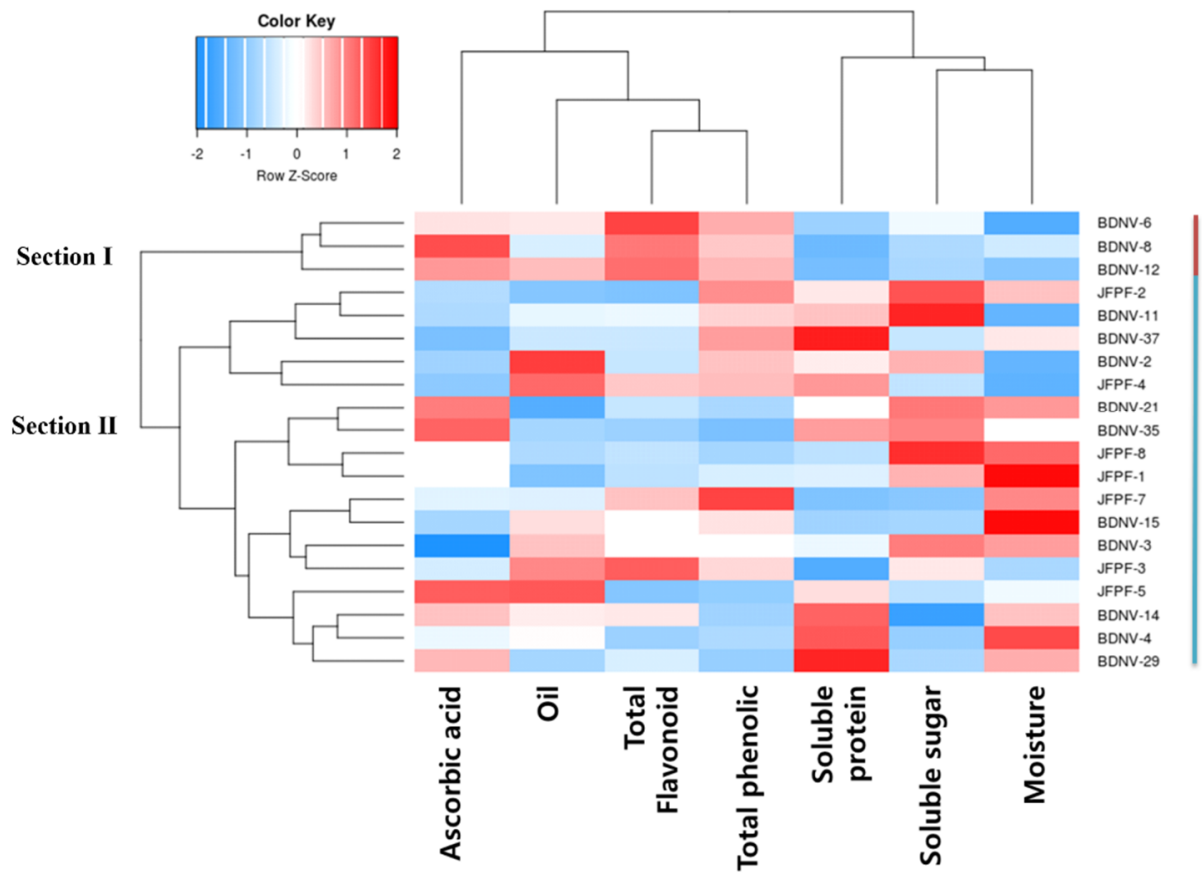

Figure 2. Cluster analysis of 20 avocado accessions based on seven quality traits. The red and blue highlights correspond to high and low contents. The z score indicates quality traits values. BDNV, avocado accessions that originated from Baodao New Village; JFPF, avocado accessions that originated from the Jianfeng private farm.

\subsection{Molecular Characterization}

Four accessions (JFPF-1, JFPF-2, JFPF-3, and JFPF-4) that originated from the Jianfeng private farm and four accessions (BDNV-2, BDNV-3, BDNV-4, and BDNV-8) that originated from Baodao New Village representing different morphotypes were analyzed with 16 EST-SSR markers (Figure 1). Of these markers, 10 were selected according to band clarity, polymorphism, and amplification stability to analyze the genetic diversity of 56 avocado accessions. The fragment size ranged from 121 to $271 \mathrm{bp}$. The 10 markers yielded a total of 31 fragments (ranging from 2 to 4), with an average of 3.1 fragments (Table 2; Table S4). We also detected two accession-specific alleles with two EST-SSR markers. One of these alleles (121 bp), which was revealed with Pa-eSSR-13, was present only in accession BDNV-14, whereas the other allele, which was detected with Pa-eSSR-14, was specific to accession BDNV-29.

The application of 10 polymorphic EST-SSRs to evaluate diversity parameters (Table 2) indicated that the Na amplified per SSR locus ranged from 2 to 4 (mean of 3.10), the Ne ranged from 1.13 to 2.64 (mean of 1.70), and the Ho varied from 0.13 to 0.68 (mean of 0.36 ). Moreover, the He varied from 0.12 to 0.62 (mean of 0.36 ) and I varied from 0.23 to 1.03 (mean of 0.65 ). 
Table 2. Diversity parameters associated with 10 polymorphic expressed sequence tag and simple sequence repeat (EST-SSRs) analyzed in 56 avocado accessions.

\begin{tabular}{cccccc}
\hline Marker Name & $\mathbf{N a}^{\mathbf{1}}$ & $\mathbf{N e}^{\mathbf{2}}$ & $\mathbf{H o}^{\mathbf{3}}$ & $\mathbf{H e}^{\mathbf{4}}$ & $\mathbf{I}^{\mathbf{5}}$ \\
\hline Pa-eSSR-1 & 2 & 1.26 & 0.23 & 0.21 & 0.36 \\
Pa-eSSR-2 & 3 & 2.64 & 0.68 & 0.62 & 1.03 \\
Pa-eSSR-4 & 3 & 1.46 & 0.35 & 0.31 & 0.58 \\
Pa-eSSR-5 & 4 & 2.04 & 0.68 & 0.51 & 0.90 \\
Pa-eSSR-7 & 3 & 1.90 & 0.24 & 0.47 & 0.81 \\
Pa-eSSR-9 & 4 & 1.46 & 0.27 & 0.31 & 0.66 \\
Pa-eSSR-12 & 2 & 1.13 & 0.13 & 0.12 & 0.23 \\
Pa-eSSR-13 & 4 & 1.96 & 0.63 & 0.49 & 0.92 \\
Pa-eSSR-14 & 3 & 1.14 & 0.13 & 0.12 & 0.27 \\
Pa-eSSR-15 & 3 & 2.03 & 0.59 & 0.51 & 0.74 \\
Total & 31 & & & & \\
Mean & 3.10 & 1.70 & 0.39 & 0.36 & 0.65 \\
\hline
\end{tabular}

${ }^{1}$ Number of observed alleles, ${ }^{2}$ effective number of alleles, ${ }^{3}$ observed heterozygosity, ${ }^{4}$ expected heterozygosity, and ${ }^{5}$ Shannon's information index.

The EST-SSR analysis revealed that the genetic similarity coefficients among all individuals ranged from 0.67 to 1.00. Similar to the cluster analysis based on quality traits, the UPGMA analysis grouped the populations into two major sections, with a similarity coefficient of 0.67 (Figure 3). In Section I, nine accessions that originated from Baodao New Village and two that originated from the Jianfeng private farm were grouped together. Section II comprised 37 and 8 accessions that originated from Baodao New Village and the Jianfeng private farm, respectively. Figure S2 presents the distribution of the first two principal coordinates of a PCoA for 56 avocado accessions. On the basis of the first principal coordinate, which accounted for $18.52 \%$ of the total variation, the accessions were clearly distributed into two groups, which generally agreed with the results of the UPGMA analysis (Figure 3). The second principal coordinate accounted for $15.74 \%$ of the total variation. Finally, we confirmed that the accessions were generally not grouped by geographical origin.

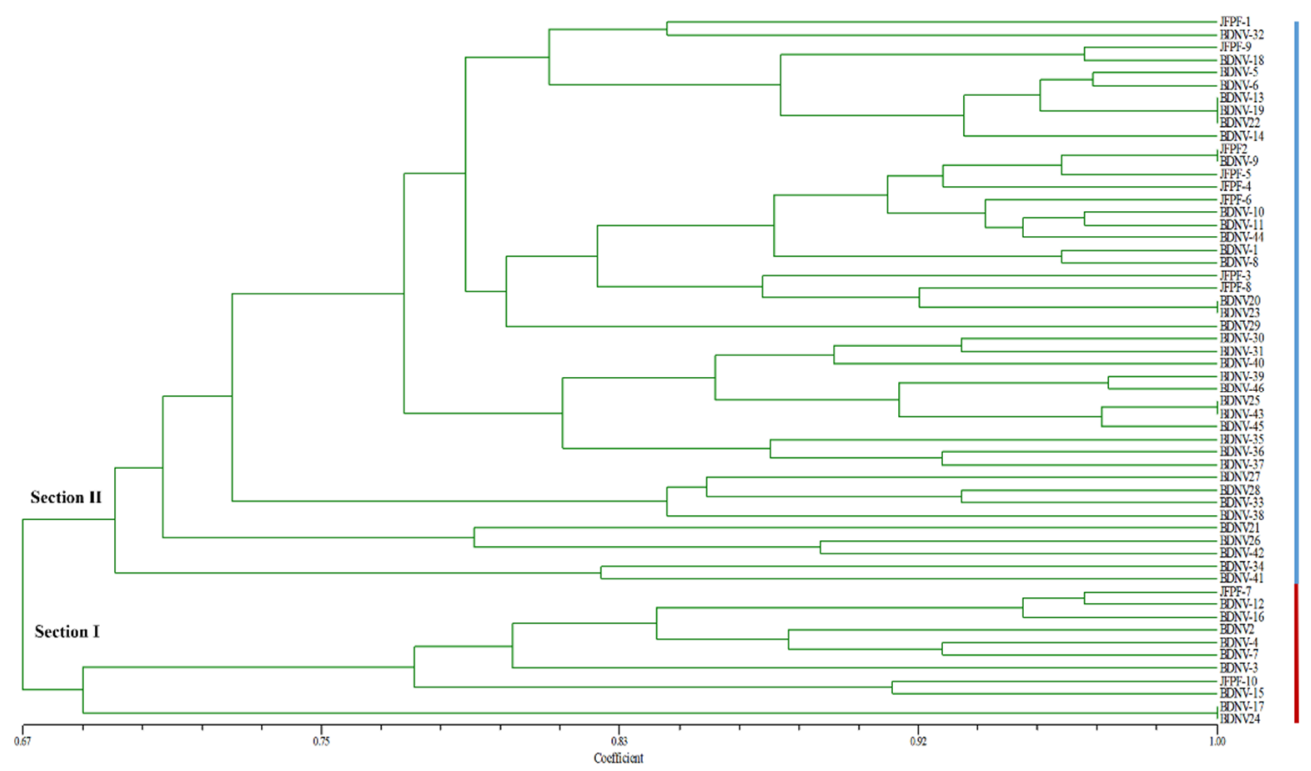

Figure 3. Dendrogram revealing the phylogenetic relationships among 56 avocado accessions based on the shared alleles for 10 EST-SSR markers. BDNV, avocado accessions that originated from Baodao New Village; JFPF, avocado accessions that originated from the Jianfeng private farm. 


\section{Discussion}

Avocado is becoming more popular in China, where it is widely cultivated and extensively used in several southern provinces, including Hainan, Yunnan, Guizhou, Guangdong, Fujian, and Taiwan [11]. These provinces are tropical and subtropical regions with long days and sufficient sunlight. Additionally, the cultural conditions and traditional agricultural practices are suitable for cultivating avocado [11]. We previously revealed the relatively high genetic diversity of the native avocado accessions from various state farms and other national scientific research units in Hainan, Yunnan, and Guizhou provinces [12]. In the present study, local avocado germplasm obtained from a private courtyard and private farm in Hainan province were included in a supplementary investigation to comprehensively clarify the genetic relationships among native avocado germplasm in China, with implications for improving avocado breeding programs.

The accurate determination of avocado accession races may ensure that avocado germplasm is effectively exploited by researchers, professional plant breeders, and farmers worldwide [7]. In the current study, we applied six avocado race-specific KASP markers to confirm the races of 56 native avocado accessions collected from two sites in Hainan province (Baodao New Village and the Jianfeng private farm). Interestingly, the KASP genotyping results revealed that all 56 native avocado accessions were complex hybrids (Guatemalan $\times$ Mexican $\times$ West Indian hybrids), which differed from the races assigned to the native avocado accessions collected from two other sites in Hainan province [12]. The reason for the discrepancy between studies may be related to the natural hybridization between high-quality foreign Guatemalan $\times$ West Indian and Guatemalan $\times$ Mexican hybrids introduced by CATAS researchers. In this study, 46 native avocado accessions were collected from Baodao New Village, which is where the CATAS headquarters is located. Many high-quality foreign Guatemalan $\times$ West Indian and Guatemalan $\times$ Mexican hybrids from the CATAS-affiliated avocado germplasm resource garden have been cultivated for human consumption and have been grown in the private courtyards of CATAS farmers and staff in Baodao New Village. Therefore, it is likely that natural hybridizations often occurred between these foreign hybrids in Baodao New Village because of a lack of strict cultivation management practices, and the high-quality offspring (i.e., Guatemalan $\times$ Mexican $\times$ West Indian hybrids) gradually developed and remained. In contrast, the CATAS avocado germplasm resource garden and the Daling State Farm were strictly managed by the government regarding the cultivation of imported avocado germplasm, thereby eliminating natural hybridizations [12].

The results of the UPGMA, PCA, and PCoA analyses in this study indicated that the examined native avocado accessions did not cluster together based on geographical origins. In addition, lower values were also found regarding the similarity coefficient of UPGMA and total variation of PCA and PCoA based EST-SSR and quality traits analyses. These results may have been at least partly due to the relatively short distance separating Baodao New Village and the Jianfeng private farm $(<200 \mathrm{~km})$, which facilitated the flow of avocado germplasm within regions and may have contributed to the similar genetic backgrounds of the avocado germplasm.

In the current study, lower values were found regarding the similarity coefficient of UPGMA and total variation of PCA and PCoA based EST-SSR and quality traits analyses, which hamper distinguishing the genetic diversity of avocado landraces from China. For characterization of quality traits for genetic diversity assessment, we intend to enlarge the number of native avocado accessions from 20 to 56 when all 56 native avocado accessions in this study bear fruit. In addition, we will add morphological characters and other quality traits along with seven quality traits measured in this study to assess the genetic diversity again. For molecular characterization for genetic diversity assessment, we will replace EST-SSR analysis by high-throughput genome-scale sequencing technologies (such as genome resequencing and specific length amplified fragment sequencing), in which a large amount of SNP data will improve greatly genetic diversity assessment.

In this study, the average Ne for the EST-SSR markers was 3.10, which was lower than the average number of alleles per locus (4.73 to 18.8) reported in earlier investigations $[8,12,15,16,22,27]$. In our two previous studies regarding the genetic diversity of avocado populations, one avocado population 
included seven foreign commercial cultivars and 39 native accessions collected from five gardens in three provinces in China, with 4.73 EST-SSR alleles per locus [12], whereas the other avocado population comprised 20 foreign commercial cultivars and eight native accessions collected from three gardens in three provinces in China, with 6.13 EST-SSR alleles per locus [22]. Accordingly, the 56 avocado accessions in the present study are likely not only genotypically the same, they may also be native avocado accessions that gradually developed in relatively isolated regions in the same province. Flavonoid content, in the current study, possessed the highest coefficients of variation in 20 native avocado accessions compared to the other six quality traits, and finally, the native avocado accessions were generally grouped according to their total flavonoid content rather than their geographical origin. In our previous study, flavonoid contents of the 16 native avocado accessions collected from three provinces in China were measured, and the difference in flavonoid contents of the 16 native avocado accessions reached nearly 113 times [28]. Besides, the significant differences in flavonoid content were observed among most of the accessions $(p<0.05)$ [28]. These results suggested that flavonoid content could be referred to as an indicative biochemical marker in distinguishing avocado accessions. Similarly, Donetti and Terry also suggested oleic acid as a potential biochemical marker in defining growing area and ripening stage of imported avocado fruit cv. Hass [29].

\section{Conclusions}

In this study, 56 native avocado accessions, including 46 originating from Baodao New Village and 10 originating from the Jianfeng private farm, were examined to clarify their races and genetic diversity. The results of a KASP genotyping analysis with six race-specific KASP markers indicated that all 56 avocado accessions were Guatemalan $\times$ Mexican $\times$ West Indian hybrids. Additionally, differences in the total flavonoid contents enabled the quality trait-based differentiation of native avocado accessions. The UPGMA, PCA, and PCoA analyses of seven quality traits and 10 EST-SSR markers resulted in the similar grouping of avocado accessions. Moreover, this grouping was not according to geographical origins. Thus, the combined analysis of molecular characteristics and quality traits was useful for elucidating the genetic diversity among native avocado landraces in Hainan province, China. The information regarding the diversity of this germplasm presented herein may be useful for breeding new avocado varieties and for improving the management of large collections of avocado species.

Supplementary Materials: The following are available online at http://www.mdpi.com/2077-0472/10/4/102/s1, Table S1: Details regarding the KASP primers; Table S2: Racial assignment of 56 avocado accessions; Table S3. Summary of seven quality traits in 56 avocado accessions; Table S4: Summary of 10 EST-SSRs in 56 avocado accessions; Figure S1: Principal component analysis of 20 avocado accessions based on seven quality traits. BDNV, avocado accessions that originated from Baodao New Village; JFPF, avocado accessions that originated from the Jianfeng private farm; Figure S2: Principal coordinate analysis of 56 avocado accessions based on 10 EST-SSR markers. BDNV, avocado accessions that originated from Baodao New Village; JFPF, avocado accessions that originated from the Jianfeng private farm.

Author Contributions: Y.G., R.Z., and W.M. conceived and designed the experiments; Y.L. (Yuanzheng Liu). performed the experiments; X.L. analyzed the data; Y.L. (Yanxia Li) and Y.Y. helped complete the experiments; X.Z. contributed materials; and Y.G. wrote the manuscript. All authors have read and agreed to the published version of the manuscript.

Funding: This research was funded by the Natural Science Foundation of Hainan Province of China (grant number 319QN266, 2019RC264), the Integrated demonstration of key techniques for the industrial development of featured crops in rocky desertification areas of Yunnan-Guangxi-Guizhou provinces (grant number SMH2019-2021), the Ministry of agriculture and rural affairs hot technology pilot demonstration project (grant number 20203578), the Ministry of agriculture and rural affairs agricultural variety improvement special funds (grant number 20201982), and the earmarked fund for the belt and road tropical project (BARTP-07).

Acknowledgments: We thank William Yajima for editing the English text of a draft of this manuscript.

Conflicts of Interest: The authors declare no conflict of interest. 


\section{References}

1. Kuhn, D.N.; Groh, A.; Rahaman, J.; Freeman, B.; Arpaia, M.L.; Van den Berg, N.; Abeysekara, N.; Manosalva, P.; Chambers, A.H. Creation of an avocado unambiguous genotype SNP database for germplasm curation and as an aid to breeders. Tree Genet. Genomes 2019, 15, 71. [CrossRef]

2. Rendón-Anaya, M.; Ibarra-Laclette, E.; Méndez-Bravo, A.; Lan, T.Y.; Zheng, C.F.; Carretero-Paulet, L.; Perez-Torres, C.A.; Chacón-López, A.; Hernandez-Guzmán, G.; Chang, T.H. The avocado genome informs deep angiosperm phylogeny, highlights introgressive hybridization, and reveals pathogen-influenced gene space adaptation. Proc. Natl. Acad. Sci. USA 2019, 116, 17081-17089. [CrossRef] [PubMed]

3. Ge, Y.; Cheng, Z.H.; Si, X.Y.; Ma, W.H.; Tan, L.; Zang, X.P.; Wu, B.; Xu, Z.N.; Wang, N.; Zhou, Z.X.; et al. Transcriptome profiling provides insight into the genes in carotenoid biosynthesis during the mesocarp and seed developmental stages of avocado (Persea americana). Int. J. Mol. Sci. 2019, 20, 4117. [CrossRef] [PubMed]

4. Ge, Y.; Dong, X.S.; Wu, B.; Wang, N.; Chen, D.; Chen, H.H.; Zou, M.H.; Xu, Z.N.; Tan, L.; Zhan, R.L. Evolutionary analysis of six chloroplast genomes from three Persea americana ecological races: Insights into sequence divergences and phylogenetic relationships. PLOS ONE 2019, 14, e0221827. [CrossRef]

5. Galindo-Tovar, M.E.; Ogata-Aguilar, N.; Arzate-Fernandez, A.M. Some aspects of avocado (Persea americana Mill.) diversity and domestication in Mesoamerica. Genet. Resour. Crop Evol. 2008, 55, 441-450. [CrossRef]

6. Talavera, A.; Soorni, A.; Bombarely, A.; Matas, A.J.; Hormaza, J.I. Genome-Wide SNP discovery and genomic characterization in avocado (Persea americana Mill.). Sci. Rep. 2019, 9, 20137. [CrossRef]

7. Schaffer, B.; Wolstenholme, B.N.; Whiley, A.W. The Avocado: Botany, Production and Uses, 2nd ed.; CPI Group (UK) Ltd: Croydon, UK, 2012.

8. Gross-German, E.; Viruel, M.A. Molecular characterization of avocado germplasm with a new set of SSR and EST-SSR markers: Genetic diversity, population structure, and identification of race-specific markers in a group of cultivated genotypes. Tree Genet. Genomes 2013, 9, 539-555. [CrossRef]

9. Ge, Y.; Hu, F.C.; Tan, L.; Wu, B.; Wang, T.; Zhang, T.; Ma, F.N.; Cao, J.Q.; Xu, Z.N.; Zhan, R.L. Molecular diversity in a germplasm collection of avocado accessions from the tropical and subtropical regions of China. Crop Breed. Appl. Biot. 2019, 19, 153-160. [CrossRef]

10. Zhang, L.; Zhang, D.S.; Liu, K.D. Environmental analysis and countermeasures for industrial development of Hainan avocado. Chin. J. Agric. Resou. Reg. Plan. 2015, 36, 78-84.

11. Ge, Y.; Si, X.Y.; Lin, X.E.; Wang, J.S.; Zang, X.P.; Ma, W.H. Advances in avocado (Persea americana Mill.). South China Fruit 2017, 46, 63-70. [CrossRef]

12. Ge, Y.; Zang, X.P.; Tan, L.; Wang, J.S.; Liu, Y.Z.; Li, Y.X.; Wang, N.; Chen, D.; Zhan, R.L.; Ma, W.H. Single-molecule long-read sequencing of avocado generates microsatellite markers for analyzing the genetic diversity in avocado germplasm. Agronomy 2019, 9, 512. [CrossRef]

13. Fiedler, J.; Bufler, G.; Bangerth, F. Genetic relationships of avocado (Persea americana Mill.) using RAPD markers. Euphytica 1998, 101, 249-255. [CrossRef]

14. Furnier, G.R.; Cummings, M.P.; Clegg, M.T. Evolution of the avocados as revealed by DNA restriction site variation. J. Hered. 1990, 81, 183-188. [CrossRef]

15. Ashworth, V.E.T.M.; Clegg, M.T. Microsatellite markers in avocado (Persea americana Mill.) genealogical relationships among cultivated avocado genotypes. J. Hered. 2003, 94, 407-415. [CrossRef]

16. Schnell, R.J.; Brown, J.S.; Olano, C.T.; Power, E.J.; Krol, C.A.; Kuhn, D.N.; Motamayor, J.C. Evaluation of avocado germplasm using microsatellite markers. J. Amer. Soc. Hort. Sci. 2003, 128, 881-889. [CrossRef]

17. Chen, H.; Morrel, P.L.; Ashwoth, V.E.T.M.; De la Cruz, M.; Clegg, M.T. Nucleotide diversity and linkage disequilibrium in wild avocado (Persea americana Mill.). J. Hered. 2008, 99, 382-389. [CrossRef]

18. Chen, H.; Morrel, P.L.; Ashwoth, V.E.T.M.; De la Cruz, M.; Clegg, M.T. Tracing the geographic origins of mayor avocado cultivars. J. Hered. 2009, 100, 56-65. [CrossRef]

19. Liu, C.; Ge, Y.; Wang, D.J.; Li, X.; Yang, X.X.; Cui, C.S.; Qu, S.P. Morphological and molecular diversity in a germplasm collection of seed pumpkin. Sci. Hortic. 2013, 154, 8-16. [CrossRef]

20. Ramírez, I.M.; Fuentes, J.L.; Rodríguez, N.N.; Coto, O.; Cueto, J.; Becker, D.; Rohde, W. Diversity analysis of Cuban avocado varieties based on agro-morphological traits and DNA polymorphisms. J. Genet. Breed. 2005, 59, 241-252.

21. Ge, Y.; Zhang, T.; Wu, B.; Tan, L.; Ma, F.; Zou, M.; Chen, H.; Pei, J.; Liu, Y.; Chen, Z.; et al. Genome-wide assessment of avocado germplasm determined from specific length amplified fragment sequencing and 
transcriptomes: Population structure, genetic diversity, identification, and application of race-specific markers. Genes 2019, 10, 215. [CrossRef]

22. Ge, Y.; Tan, L.; Wu, B.; Wang, T.; Zhang, T.; Chen, H.; Zou, M.; Ma, F.; Xu, Z.; Zhan, R. Transcriptome sequencing of difference avocado ecotypes: De novo transcriptome assembly, annotation, identification and validation of EST-SSR markers. Forests 2019, 10, 411. [CrossRef]

23. Ge, Y.; Ramchiary, N.; Wang, T.; Liang, C.; Wang, N.; Wang, Z.; Choi, S.R.; Lim, Y.P.; Piao, Z.Y. Development and linkage mapping of unigene-derived microsatellite markers in Brassica rapaL. Breed. Sci. 2011, 61, 160-167. [CrossRef]

24. Ge, Y.; Si, X.Y.; Cao, J.Q.; Zhou, Z.X.; Wang, W.L.; Ma, W.H. Morphological characteristics, nutritional quality, and bioactive constituents in fruits of two avocado (Persea americana) varieties from hainan province, China. J. Agric. Sci. 2017, 9, 8-17. [CrossRef]

25. Krawczak, M.; Nikolaus, S.; von Eberstein, H.; Croucher, P.J.; El Mokhtari, N.E.; Schreiber, S. PopGen: Population based recruitment of patients and controls for the analysis of complex genotype-phenotype relationships. Community Genet. 2006, 9, 55-61. [CrossRef]

26. Rohlf, F.J. NTSYS pc: Numerical Taxonomy and Multivariate Analysis System; Version 2.1; Exeter Software: New York, NY, USA, 2000.

27. Alcaraz, M.L.; Hormaza, J.I. Molecular characterization and genetic diversity in an avocado collection of cultivars and local Spanish genotypes using SSRs. Hereditas 2007, 144, 244-253. [CrossRef]

28. Ge, Y.; Si, X.Y.; Cao, J.Q.; Hu, F.C.; Wu, K.; Xu, H.M.; Tan, H.R.; Zang, X.P.; Ma, W.H. The analysis on the nutrition and biological activity of avocado fruit. South China Fruits 2017, 46, 50-56. [CrossRef]

29. Donetti, M.; Terry, L.A. Biochemical markers defining growing area and ripening stage of imported avocado fruit cv. Hass. J. Food Compos. Anal. 2014, 34, 90-98. [CrossRef]

(C) 2020 by the authors. Licensee MDPI, Basel, Switzerland. This article is an open access article distributed under the terms and conditions of the Creative Commons Attribution (CC BY) license (http://creativecommons.org/licenses/by/4.0/). 